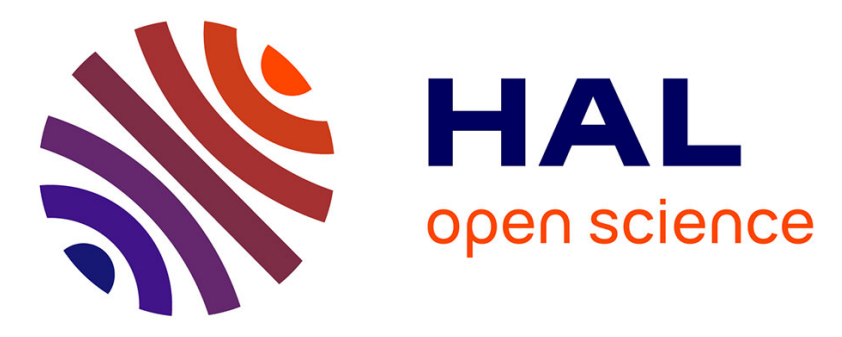

\title{
Absolute self-calibrated room-temperature terahertz powermeter
}

Christophe Pradère, Jean-Pascal Caumes, Jean Toutain, Emmanuel Abraham, Bruno Chassagne, Jean-Christophe Batsale

\section{To cite this version:}

Christophe Pradère, Jean-Pascal Caumes, Jean Toutain, Emmanuel Abraham, Bruno Chassagne, et al. Absolute self-calibrated room-temperature terahertz powermeter. Applied optics, 2013, 52 (11), pp.2320-2324. 10.1364/AO.52.002320 . hal-00812181

\section{HAL Id: hal-00812181 \\ https://hal.science/hal-00812181}

Submitted on 8 Mar 2018

HAL is a multi-disciplinary open access archive for the deposit and dissemination of scientific research documents, whether they are published or not. The documents may come from teaching and research institutions in France or abroad, or from public or private research centers.
L'archive ouverte pluridisciplinaire HAL, est destinée au dépôt et à la diffusion de documents scientifiques de niveau recherche, publiés ou non, émanant des établissements d'enseignement et de recherche français ou étrangers, des laboratoires publics ou privés.

\section{(1)(1) $\$(0)$}

Distributed under a Creative Commons Attribution - NonCommercial - ShareAlikel 4.0 


\title{
Absolute self-calibrated room-temperature terahertz powermeter
}

\author{
Christophe Pradere, ${ }^{1, *}$ Jean-Pascal Caumes, ${ }^{1}$ Jean Toutain, ${ }^{1}$ Emmanuel Abraham, ${ }^{2}$ \\ Bruno Chassagne,$^{3}$ and Jean-Christophe Batsale ${ }^{1}$ \\ 'I2M TREFLE department, UMR CNRS 5295, Esplanade des Arts et Métiers, Talence 33405, France \\ ${ }^{2}$ LOMA, Université de Bordeaux, CNRS, UMR 5798, Talence 33405, France \\ ${ }^{3}$ ALPhANOV, Technological Laser and Optical Centre, 351 Cours de la Libération, Talence 33405, France \\ *Corresponding author: christophe.pradere@ensam.eu
}

\begin{abstract}
Coupling optical and thermal properties of a terahertz $(\mathrm{THz})$ thermal converter based on the Seebeck effect provides an unsupplied room temperature measuring device dedicated to $\mathrm{THz}$ power metrology. Performance characteristics such as broadband response $(030 \mathrm{THz})$, high sensitivity $\left(<25 \mu \mathrm{W} \cdot \mathrm{Hz}^{-0.5}\right)$, and the possibility to develop an internal absolute self calibration estimated at $9.93 \mathrm{~W} \cdot \mathrm{V}^{-1}$ are reported. Advantages and drawbacks of this $\mathrm{THz}$ powermeter are discussed.
\end{abstract}

OCIS codes: $\quad 040.5150,040.1880$.

Since the potential of terahertz $(\mathrm{THz})$ technology has been highlighted by its various applications in such large domains as security, biology, medicine, and nondestructive inspection, a great need exists for room-temperature efficient and wide-spectral-band $\mathrm{THz}$ sources (i.e., $30-3000 \mu \mathrm{m}$ wavelength). Complementary to the development of such sources, extension of the low-temperature bolometer sensitivity has been largely achieved from infrared (IR) to $\mathrm{THz}$ frequencies [1]. Several promising $\mathrm{THz}$ detection techniques have hence emerged based on resonant excitation of a quartz tuning fork [2] or using a pressure transducer [3]. Despite this, the development of a more efficient $\overline{\mathrm{TH}} \mathrm{Hz}$ detector has not yet reached a technological maturity state, particularly for absolute power quantification. In this context, our proposed patented physical system consists in using a $\mathrm{THz}$ to thermal converter (TTC) in order to efficiently absorb an incident $\mathrm{THz}$ radiation. The TTC temperature elevation induces a heat flux that could be precisely quantified using a contact thermal flux sensor. This technique has performed sensitive enthalpy measurements of chemical reactions in a microfluidic device [4]. It has also been applied for electromagnetic-wave imaging using thermal plate temperature measurements by IR microbolometer cameras [5]. Hence, measuring the TTC thermal flux instead of its temperature elevation is the key point in order to realize sensitive power measurements of $\mathrm{THz}$ power.

In this paper, a new room-temperature $\mathrm{THz}$ detector scheme is presented, demonstrating several important properties, such as broadband response, high sensitivity, and the possibility of developing an internal absolute calibration. Compared to commercially available bolometers or Golay cells, our low-cost solution is fully integrated and does not require any external supply voltage.

The schematic of our system is detailed in Fig. 1 . The multilayered thermal system is composed of a $5 \mathrm{~mm}$ thick high-density polyethylene (HDPE) insulator window and a TTC (a $30 \mu \mathrm{m}$ thick carbon-doped paper [6,7]) in contact with a $3.2 \mathrm{~mm}$ thick thermopile flux sensor (Melcor Peltier module CP1.4-127-06L with differential voltage acquired using a microvoltmeter Agilent 3140A). A mechanical symmetry 


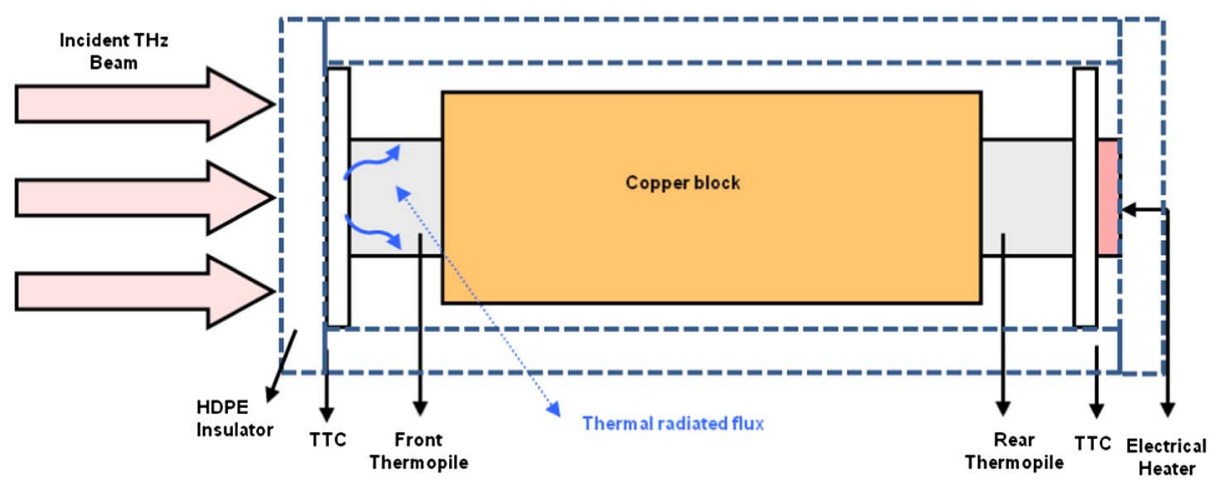

Fig. 1. (Color online) Scheme of the room temperature THz detector. Front face: THz detection using a TTC placed on a thermopile behind an HDPE window (dotted squared). Rear face: in situ calibration using an electrical heater (see text for details). The semi infinite medium copper block minimizes flux transfer from front to rear sides.

(insulator, TTC, and a second thermopile) is designed at the rear face for the electrical power calibration setup using a thin resistive electrical heater. Both thermopiles are separated by a high-mass-density $20 \mathrm{~mm}$ long copper block.

Several aspects in this design are fundamental to perform an absolute powermeter calibration, meaning optimization of the $\mathrm{THz}$ absorption and heat flux detection. First, to avoid thermal losses away from the two thermopiles and along the copper block, the whole system was thermally isolated from the surrounding using an insulated $5 \mathrm{~mm}$ thick HDPE. To improve the detector sensitivity, we used two differential Peltier systems to minimize the surrounding roomtemperature long time variation. Second, it is fundamental to precisely characterize the optical and thermal parameters of each material together with the heat flux (TTC, insulators, copper). An absorption coefficient of $200 \mathrm{~cm}^{1}$ at $0.1 \mathrm{THz}$ frequency has been measured for the TTC. The front sensor system aims to measure the $\mathrm{THz}$ incident radiation, whereas the rear side is used for power calibration. This is possible because a symmetrical mechanical system has been implemented, simulating a semi-infinite thermal medium with high mass density and the specific heat of the copper. Indeed, the block temperature is considered isothermal. For that, it defines a temperature reference between the rear and the front thermopiles.

From a thermal point of view, the complete system could be modelized [8] by a multilayered scheme with an internal heat source induced by the incident $\mathrm{THz}$ radiation. Nevertheless, according to the thickness of the TTC, a simplified model is proposed here. For that, we assumed that the thermal behavior of the TTC could be represented by a thin body model with internal heat sources (due to incident $\mathrm{THz}$ radiation or electrical heater) and that the heat transfer from the TTC to the brass bulk is characterized by a thermal resistance acting as thermal heat losses. Finally, we also assumed that the heat sources always have duration lower than the response time of the system given by the heat-loss characteristic time. In fact, the usual temporal form of used sources is a pulse, step, or periodic pulse, where the duration can be considered very short. From such a point of view, the transient measured voltage of the Peltier element is represented by the following equation:

$$
U(t)=U_{f}(1-\exp (-t / H)),
$$

where $U_{f}[\mathrm{~V}]$ corresponds to the final value (in steady state) of the measured voltage and $H$ [s] is the characteristic response time of the thermal heat losses. Hence, due to the Seebeck effect, the acquired voltage $U$ across the thermopile is proportional to the incident thermal heat flux $P$ [W] induced by the electrical power through the Joule effect or the incident $\mathrm{THz}$ radiation. This thermal flux is classically given by

$$
P=\alpha_{P} U \quad \text { with } \alpha_{P}=\frac{\lambda G}{\beta},
$$

where $\lambda$ is the thermal conductivity [W $\cdot \mathrm{m}^{1} \cdot \mathrm{K}^{1}$ ], $\beta$ is the Seebeck coefficient $\left[\mathrm{V} \cdot \mathrm{K}^{1}\right]$, and $G$ is the shape factor $[\mathrm{m}]$. The calibration coefficient $\alpha_{P}\left[\mathrm{~W} \cdot \mathrm{V}^{1}\right]$ corresponds to the Peltier sensitivity.

In order to self-calibrate the powermeter, it is necessary to find both the response time $H$ of the system and the calibration coefficient $\alpha_{P}$. For that, a typical experimental calibration protocol using the Joule effect is performed as illustrated in Fig. 2 . For a given electrical power in the $300 \mathrm{~mW}$ to $1.5 \mathrm{~W}$ range, we recorded the voltage $U$ versus time across the thermopile until the thermal steady state was reached. From these measurements, it is possible with the inverseprocessing method to minimize the measured value with the analytical solution given by Eq. (1) in order to find the two parameters $H$ and $\alpha_{P}$. First, the response time $H$ of the system is estimated from the four curves of Fig. 2 according to Eq. (1). The estimated values are $H_{1}=85.7 \mathrm{~s}(0.3 \mathrm{~W})$, $H_{2}=85.6 \mathrm{~s}(0.5 \mathrm{~W}), \quad H_{3}=90.2 \mathrm{~s}(0.9 \mathrm{~W}), \quad$ and $H_{4}=87.9 \mathrm{~s}(1.5 \mathrm{~W})$. From these values, the average response time of the system $H_{A}=87.3 \mathrm{~s}$ is deduced. We can notice that the standard deviation is lower than $2 \%$. This average value is in agreement with the Peltier responding time, which is approximatively given by the ratio between the Peltier thickness and the thermal diffusivity $\left(t=e^{2} / a=102 \mathrm{~s}\right.$ with $e=3.2 \mathrm{~mm}$ and $a=10^{7} \mathrm{~m}^{2} \cdot \mathrm{s}^{1}$, data given by 


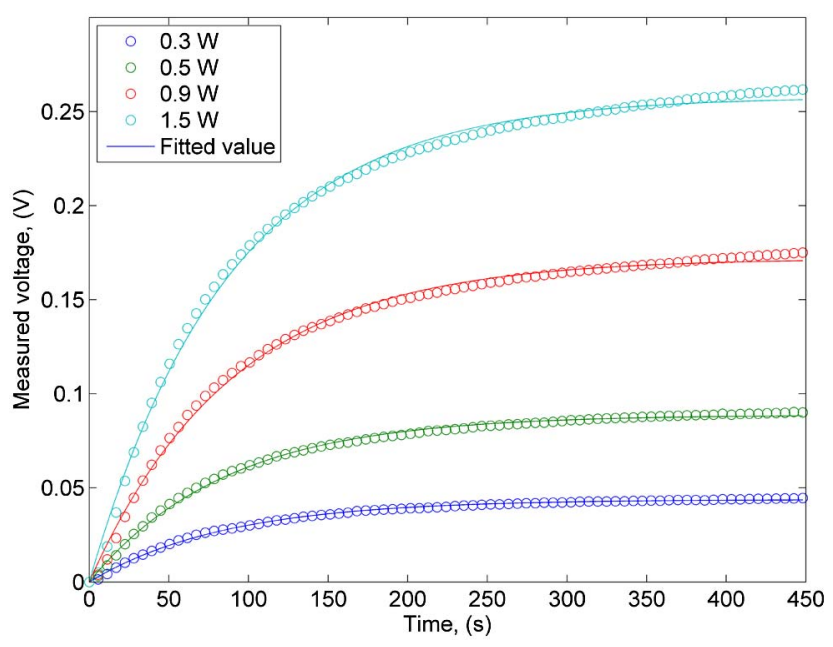

Fig. 2. (Color online) In situ Joule effect calibration: measure ment of thermopile voltage response versus time for different imposed electrical powers.

manufacturer, Melcor Peltier module CP1.4-127-06L). Then, the applied electrical powers are reported in Fig. $\underline{3}$ as a function of the final estimated values of the measured voltage $U_{f}$. This evolution is perfectly linear with a fitted slope corresponding to the calibration coefficient $\alpha_{P}=5.56 \mathrm{~W} \cdot \mathrm{V}^{1}$. Finally, from this thermal calibration and according to Eqs. (1) and (2), we obtained the total transfer function of the system, which allows us to directly estimate the power measured by the Peltier from the following formulation:

$$
P(t)=\alpha_{P} U(t)=\alpha_{P} U_{f}\left(1-\exp \left(-t / H_{A}\right)\right)
$$

with $P(t)$ [W], the power measured by the Peltier as a function of time, $H_{A}[\mathrm{~s}]$ the average estimated response time, and $\alpha_{P}\left[\mathrm{~W} \cdot \mathrm{V}^{1}\right]$ the estimated calibration

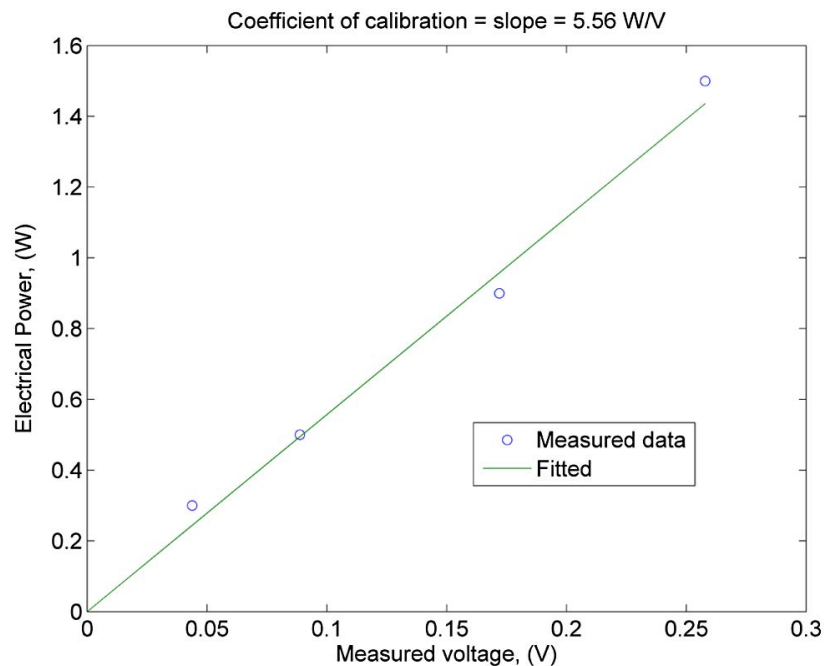

Fig. 3. (Color online) In situ Joule effect calibration: measured differential steady state voltage versus imposed electrical power fitted by linear regression giving the calibration coefficient of the system. coefficient. In order to avoid a long time drift due to external heat losses, it is possible to use the short time development of Eq. (3), available when the ratio $t / H_{A}<0.1$. In this case, an asymptotic expansion close to zero is applied to the exponential function and Eq. () can be written as

$$
\log [P(t)]=\log (t)+\log \left(\frac{\alpha_{P} U_{f}}{H_{A}}\right), \quad \text { if } t / H_{A}<0.1 .
$$

This simplified formulation exhibits the linear relation between $\log [P(t)]$ and $\log (t)$. Moreover, the $y$ intercept of the linear fit can be used in order to earlier estimate the final value $\alpha_{P} U_{f}$ of the power measured by the Peltier. With this technique, long time drift is avoided and the measurement of the response time of the system can be decreased 10 times.

Due to the mechanical and thermal symmetry of the system (Fig. 1), this calibration is also valid for the front thermopile. Hence, when a $\mathrm{THz}$ radiation illuminates the front TTC, the measured voltage is similarly converted into a heat flux using this former coefficient $\alpha_{P}$ but corrected by the interface Fresnel losses. Indeed, for an incident THz power $P_{0}$, the TTC will absorb $P_{\mathrm{TTC}}=A_{\mathrm{TTC}} P_{0}$, where $A_{\mathrm{TTC}}$ is the TTC absorption of the incident $\mathrm{THz}$ radiation. This parameter is defined as $A_{\mathrm{TTC}}=T_{1}\left(1-T_{2}-R_{2}\right)$, with the transmission $T_{1}=1-\left(1-n_{\mathrm{HDPE}}\right)^{2} /\left(1+n_{\mathrm{HDPE}}\right)^{2}$, the reflection $R_{2}=\left(n_{\mathrm{HDPE}}-n_{\mathrm{TTC}}\right)^{2} /\left(n_{\mathrm{HDPE}}+n_{\mathrm{TTC}}\right)^{2}$ calculated from for the reflection losses at the air/ insulator and insulator/TTC interfaces, and the transmission $T_{2}$ measured by time-resolved $\mathrm{THz}$ spectroscopy as shown in Fig. 4 for the 0.1-2.5 $\mathrm{THz}$ spectral range. In this spectral range, the respective refractive indexes are given by $n_{\mathrm{HDPE}}=1.5$ and $n_{\mathrm{TTC}}=2$. We found $T_{1}=0.96$, $T_{2}=0.6$ and $R_{2}=0.02$. This gives $A_{\mathrm{TTC}}=0.56$ for the $30 \mu \mathrm{m}$ thick TTC. As a result, only $56 \%$ of the incident $\mathrm{THz}$ power is converted into a heat flux in the TTC. One must note that even if we could optimize

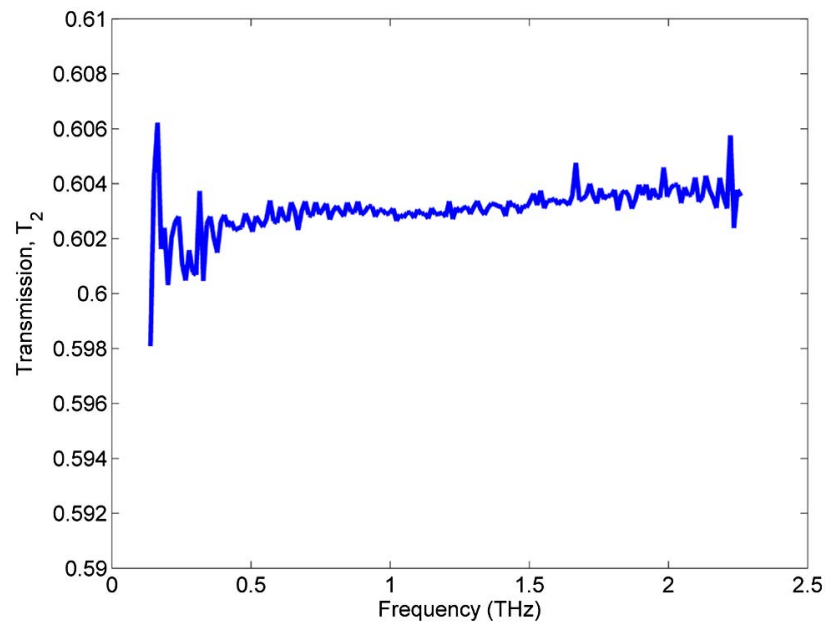

Fig. 4. (Color online) Calculated absorbance of the TTC from measured transmission using $\mathrm{THz}$ time resolved spectroscopy. 
the TTC absorption until $100 \%$, this would result in a power conversion into a heat flux of $94 \%$. This will only increase the detector performance by a factor of 1.7. Finally, we can write the relation $P_{\text {TTC }}(t)=$ $A_{\mathrm{TTC}} P_{0}(t)=\alpha_{P} U(t)$ and give the final expression of the measured power $P_{0}(t)$ of the incident $\mathrm{THz}$ radiation directly converted from the measured voltage $U(t)$ :

$$
P_{0}(t)=k_{P-\mathrm{TTC}} U(t),
$$

where $k_{P \text {-TTC }}=\alpha_{P} / A_{\mathrm{TTC}}=9.93 \mathrm{~W} \cdot \mathrm{V}^{1}$ is the detector responsivity in the $0.1-2.5 \mathrm{THz}$ spectral range.

To illustrate the performance of the TTC detector, we illuminated it with a $0.11 \mathrm{THz}$ frequency continuous emitted Gunn diode. Figure 5 shows the time evolution of the measured power $P_{0}(t)$ and the estimated one (by using the calibration coefficient estimated in the calibration part), from the switching-on (step) of the source up to the steady-state regime reached after $400 \mathrm{~s}$. This long time stabilization is due to the heat flux time transfer through the $3.2 \mathrm{~mm}$ thick thermopile. Nevertheless, thanks to the thermal calibration and the short time method, it is possible to estimate the final power (before reaching the steady state) as a function of time in agreement with the Eq. (3) or Eq. (4). Figure 6 shows that the value of the estimated power according to Eq. (3) is unstable at short time, but becomes quite stable from $50 \mathrm{~s}$ with an average value of $P_{0}=(20.5 \pm 0.1) \mathrm{mW}$ and a standard deviation of $1 \%$ over all the time. At long time $(t>200 \mathrm{~s})$ we observed the influence of the external heat losses. To avoid such a problem the measurements are practically stopped after $150 \mathrm{~s}$. Nevertheless, by using the short time approximation of Eq. (4), the average value of the power can be estimated before $10 \mathrm{~s}$ and without any sensitivity to the long time thermal drift of the system. In this time range, the estimated average value of the final power

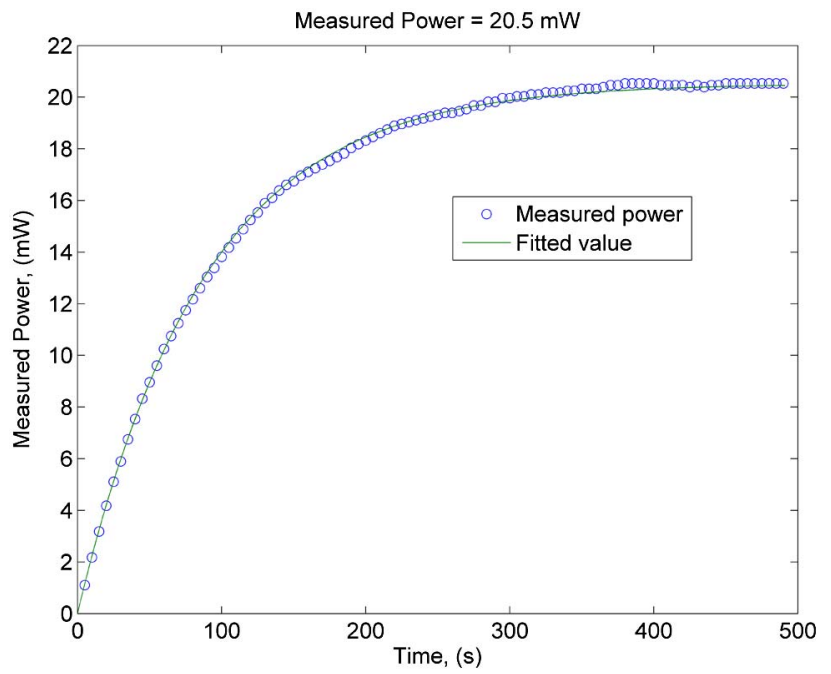

Fig. 5. (Color online) Measured power response versus acquisi tion time of a $0.11 \mathrm{THz}$ Gunn diode and fitted value using thermal modelization and inverse processing methods [Eq. (3)].

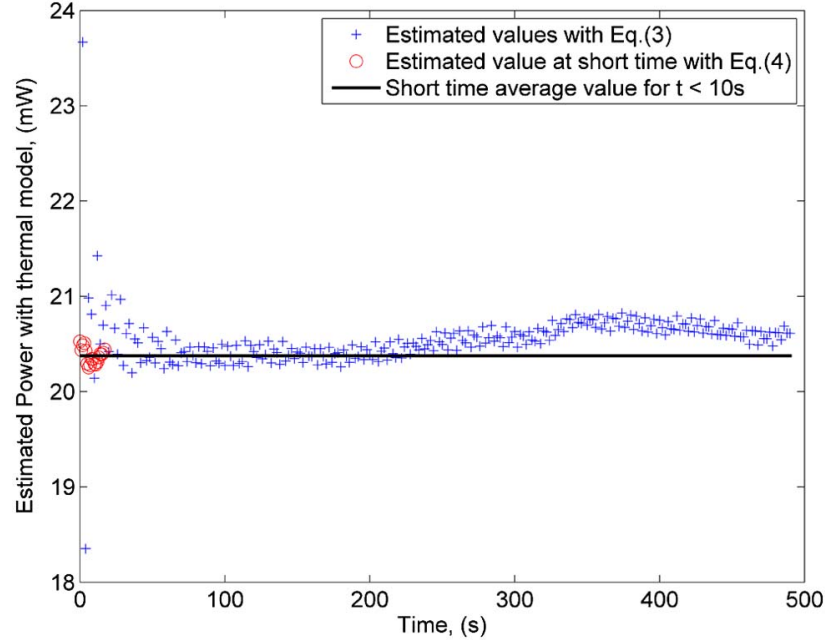

Fig. 6. (Color online) Estimated power as function of time of a $0.11 \mathrm{THz}$ Gunn diode using the thermal self calibrated model.

is equal to $P_{0}=(20.4 \pm 0.1) \mathrm{mW}$. For this $0.11 \mathrm{THz}$ Gunn diode, the obtained results are in good agreement with the $20 \mathrm{~mW}$ supplier datasheet [9]. At around $10 \mathrm{~s}$, the estimated power has an error lower than 2.5\% compared with both measured averages values. This result proves that with a good thermal calibration it is possible to obtain in real time an absolute value of the power emitted by a $\mathrm{THz}$ source in $10 \mathrm{~s}$ (only $2 \%$ of the Peltier response time). To improve this time of $10 \mathrm{~s}$, a good way is to use a thinner thermopile with lower response time (some of them could reach $1 \mathrm{~ms}$ [10]) or an IR camera.

The power metrology of different monochromatic sources largely used in the $\mathrm{THz}$ community has been tested with our TTC system. Corresponding measured powers are presented in Fig. 7. From this wide $\mathrm{THz}$ sources panel [electronic sources, backward-wave oscillator (BWO), quantum cascade laser (QCL), $\mathrm{CO}_{2}$ laser] covering a large bandwidth spectral domain $(0.1-30 \mathrm{THz})$, a high dynamical power range from 0.02 to $60 \mathrm{~mW}$ is demonstrated (power measurement error $<5 \%$ ).

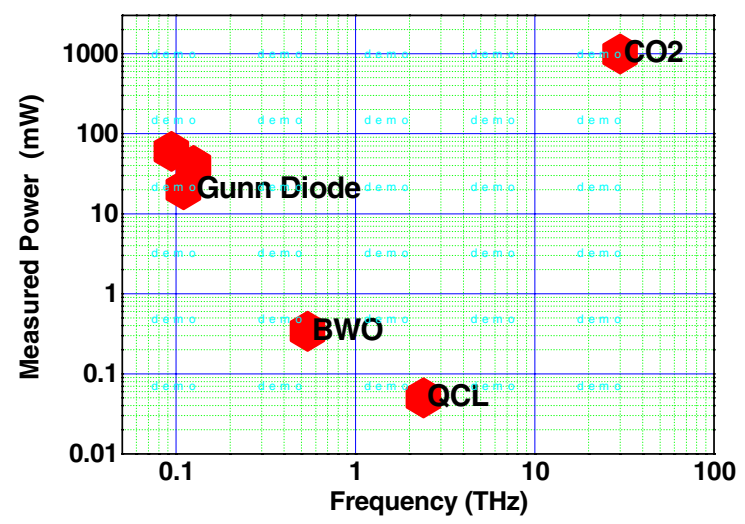

Fig. 7. (Color online) Experimental results obtained for various $\mathrm{THz}$ sources going from 0.1 to $30 \mathrm{THz}$ with power ranging from 0.05 to $1000 \mathrm{~mW}$. 
To fully characterize the performances of the TTC, we finally evaluated its noise equivalent power (NEP). First we estimated the output noise $\sigma=$ $1 \mu \mathrm{V}$ of the detector computing the standard deviation in our background measurements. Knowing the fluxmeter responsitivity $k_{P \text {-TTC }}$, the NEP reported to a $1 \mathrm{~Hz}$ bandwidth is given by [11]

$$
\mathrm{NEP}=\mathrm{k}_{P \text {-TTC }} \sigma \sqrt{2 \pi t_{\text {acq }}},
$$

where $t_{\text {acq }}$ is the acquisition time. For $k_{P \text {-TTC }}=9.93 \mathrm{~W} \cdot \mathrm{V}^{1}$, we infer an $\mathrm{NEP}$ value of $25 \mu \mathrm{W} \cdot \mathrm{Hz}^{0.5}$ in the $0.1-2.5$ spectral range. This result compares well with other reported NEP values (photoacoustic absolute powermeter with $5 \mu \mathrm{W}$. $\mathrm{Hz}^{0.5}$ [3]). Actually, the main drawback of the TTC is the time (close to $10 \mathrm{~s}$; see Fig. 6 ) necessary to perform stable measurement of the power.

In conclusion, we presented a new absolute $\mathrm{THz}$ powermeter based on a TTC. The system combines broadband response, room-temperature operation, portability, easy integration, and high sensitivity. By using two thermal sensors, we described the possibility to provide a thermal calibration using an electrical heater. This versatile and cheap device is suited to characterize $\mathrm{THz}$ and sub-THz monochromatic sources (electronic diodes, BWO, QCLs, and molecular lasers), bringing an added value for $\mathrm{THz}$ metrology. In a next step, we will study the potential of the TTC powermeter for the characterization of pulsed and broadband $\mathrm{THz}$ sources generated from photoconductive antennas or nonlinear crystals. Another improvement will be to find a new thermopile with higher time response.

\section{References}

1. A. J. Kreisler and A. Gaugue, "Recent progress in high temperature superconductor bolometric detectors: from the mid infrared to the far infrared (THz) range," Supercond. Sci. Technol. 13, 12351245 (2000).

2. U. Willer, A. Pohlkötter, W. Schade, J. Xu, T. Losco, R. P. Green, A. Tredicucci, H. E. Beere, and D. A. Ritchie, "Resonant tuning fork detector for THz radiation," Opt. Express 17, 1406914074 (2009).

3. Thomas Keating, THz power meter, http://www.terahertz.co.uk/.

4. C. Pradere, J. P. Caumes, B. Chassagne, and J. C. Batsale, French patent FR0952097 (01 April 2009).

5. C. Hany, H. Lebrun, C. Pradere, J. Toutain, and J. C. Batsale, "Thermal analysis of chemical reaction with a continuous mi crofluidic calorimeter," Chem. Eng. J. 160, 814822 (2010).

6. G. N. Kulipanov, N. G. Gavrilov, B. A. Knyazev, E. I. Kolobanov, V. V. Kotenkov, V. V. Kubarev, A. N. Matveenko, L. E. Medvedev, S. V. Miginsky, L. A. Mironenko, V. K. Ovchar, V. M. Popik, T. V. Salikova, M. A. Scheglov, S. S. Serednyakov, O. A. Shevchenko, A. N. Skrinsky, V. G. Tcheskidov, and N. A. Vinokurov, "Research highlights from the Novosibirsk 400 W average power THz FEL," Terahertz Sci. Technol. 1, 107125 (2008).

7. D. Balageas and P. Levesque, "A photothermal tool for electro magnetic phenomena characterization," Rev. Gén. Therm. 37, 725739 (1998).

8. J. Pailhes, C. Pradere, J. Toutain, J. L. Battaglia, A. Kusiak, W. Aregba, and J. C. Batsale, "Thermal quadrupole method with internal heat sources," Int. J. Thermal Sci. 53, 4955 (2012).

9. Gunn Oscillators, ZAX Millimeter Wave Corporation, www millimeterwave.com

10. Micropelt manufacturer, http://micropelt.com/.

11. J. P. Caumes, B. Chassagne, D. Coquillat, F. Teppe, and W. Knap, "Focal plane micro bolometer arrays for $0.5 \mathrm{THz}$ spatial room temperature imaging," Electron. Lett. 45, 3435 (2009). 